\title{
ANÁLISIS DE LAS PREFERENCIAS DE LOS CONSUMIDORES POR PRODUCTOS ALIMENTARIOS CON ATRIBUTOS RELATIVOS A LA BIOECONOMÍA CIRCULAR
}

\author{
$\underline{\text { Anastasio J. Villanueva }}^{a, d, *}$, Adriana Bertuglia ${ }^{a}$, Angel Perni ${ }^{c}$, M $^{\text {a }}$ del Mar Cátedra-Cerón ${ }^{b}$, Samir

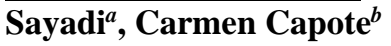 \\ a IFAPA-Instituto de Investigación y Formación Agraria y Pesquera, Área de Economía de la Cadena \\ Alimentaria (Granada, anastasioj.villanueva@juntadeandalucia.es, \\ adriana.bertuglia@juntadeandalucia.es,samir.sayadi@juntadeandalucia.es) \\ $b$ CAGPDS-Consejería de Agricultura, Ganadería, Pesca y Desarrollo Sostenible (Sevilla, \\ mariam.catedra@juntadeandalucia.es, Carmen.capote@juntadeandalucia.es) \\ ${ }^{c}$ Dpto. Economía General, Universidad de Cádiz (Cádiz, angel.perni@uca.es) \\ ${ }^{d}$ WEARE-Water, Environmental, and Agricultural Resources Economics, Universidad de Córdoba
}

\section{Resumen}

El desarrollo de la bioeconomía circular (BEC) en España pasa necesariamente por que el sector agroalimentario adopte sus principios, haciendo de este un sector económica, social y ambientalmente sostenible en el largo plazo. Resulta especialmente relevante conocer las preferencias del consumidor para poder incorporar a los productos alimentarios atributos relacionados con la BEC que son a su vez demandados por los consumidores. El presente estudio analiza las preferencias de los consumidores por productos alimentarios que incluyen dichos atributos en su etiquetado, para el caso de estudio de tomate de ensalada en Andalucía mediante una encuesta realizada en marzo 2021. En él se emplean técnicas de preferencias declaradas en una muestra representativa de un panel de 1248 consumidores. Los resultados indican que los consumidores están dispuestos a pagar un sobreprecio por cualquiera de los siete atributos considerados, en todo caso dentro del rango de $0,33-0,45 € / \mathrm{kg}$. De mayor a menor sobreprecio, los atributos se ordenan de la siguiente forma: "Contribución a la economía rural", "No generación de residuos plásticos", "No emitimos gases de efecto invernadero", "Cuidamos la biodiversidad", "Usamos el agua de forma sostenible", "Conservamos la fertilidad del suelo" y "No generamos residuos vegetales". Se observa asimismo cómo el sobreprecio que están dispuestos a pagar no sigue una función estrictamente aditiva de los atributos, dado que el sobreprecio que pagarían por el conjunto de atributos considerados es de 0,52 $€ / \mathrm{kg}$ de media. Estos resultados preliminares sugieren que el uso de estrategias de diferenciación en el sector del tomate podría aumentar su rentabilidad.

Palabras clave: Bioeconomía, Economía circular, Análisis de preferencias, Valoración contingente.

\section{Introducción}

La bioeconomía circular (BEC) integra los conceptos de bioeconomía (EC, 2018) y economía circular (Kirchherr et al., 2017), con la vocación de representar un modelo económico sostenible económica, social y ambientalmente. Incluye, por tanto, elementos comunes a ambos conceptos, como son mejorar el uso de los recursos y la ecoeficiencia, reducir la huella de carbono y la demanda de carbono fósil, y la valorización de los residuos (Carus y Dammer, 2018). Así, dentro del sector de la BEC se circunscriben aquellas actividades económicas con base biológica que a su vez aplican de forma consecuente los principios de la circularidad en sus procesos productivos.

Este "nuevo" sector se encuentra en una fase incipiente de desarrollo, habida cuenta de la elevada dependencia que presenta respecto del conocimiento y la innovación. Sin embargo, su expansión es deseable para el conjunto de la sociedad, no solo por la generación de riqueza y empleo (a menudo de calidad) resultante sino por servir como medio para poder hacer frente a los grandes retos de las sociedades actuales (i.e. el reto climático, la gestión de residuos, la pérdida de biodiversidad, el reto territorial -“el campo vaciado"-, entre otros). En este sentido, considerando que la generación de valor se evidenciará necesariamente a través del intercambio de productos en el mercado, resulta fundamental analizar las preferencias de los consumidores para estimar el valor asociado a los diferentes atributos conectados con estos retos.

Sin embargo, existe una escasez de estudios que analicen las preferencias de los consumidores por productos agroalimentarios que incluyan atributos relacionados con la BEC (i.e. por productos con base biológica donde se apliquen los principios productivos de la economía circular). En efecto, si bien existe una extensa bibliografía centrada en analizar las preferencias de los consumidores por alimentos ecológicos (Li y Kallas, 2021) y el ecoenvasado de alimentos (Ketelsen et al., 2020), la valoración de los atributos relacionados con la gestión de los residuos y de los recursos en los sistemas agroalimentarios ha recibido ciertamente una menor atención. 
En este contexto, el presente estudio tiene por objetivo analizar las preferencias de los consumidores por atributos relacionados con la BEC. Para ello, se emplea el método de valoración contingente a una muestra representativa de consumidores andaluces de tomate de ensalada.

\section{Metodología}

El método de valoración contingente es un técnica de preferencias declaradas ampliamente utilizada para la estimación de la disposición a pagar (DAP) de los consumidores por productos agroalimentarios (Li y Kallas, 2021). Aquí se ha empleado en una secuencia de dos preguntas para estimar el sobreprecio que el consumidor estaría dispuesto a pagar por determinados atributos de BEC incorporados al tomate de ensalada. La primera pregunta presenta una formulación simple dicotómica de aceptación o no del sobreprecio presentado de forma aleatoria de entre las siguientes opciones: $0,1 / 0,3 / 0,5 / 0,8 / 1 € / \mathrm{kg}$ de tomate, referidas aproximadamente a sobreprecios del 10/20/30/50/67\% adicional sobre el precio medio de tomate de ensalada (que según el Observatorio de Precios y Mercados de Andalucía, se sitúa en 1,5 €/kg). La segunda pregunta presenta una formulación semi-abierta, incluyendo cartón de pago y opción de elegir otra cantidad, inquiriendo sobre la DAP máxima que el consumidor estaría dispuesto a pagar por cada atributo. Una formulación secuencial de este tipo centrada en la DAP diferencial o sobreprecio ha mostrado buenos resultados en estudios previos (p. ej., Mesías Díaz et al., 2012). El Cuadro 1 se muestra una imagen de cómo se presentó la primera pregunta de valoración al entrevistado.

Cuadro 1. Ejemplo de tarjeta de elección relativa a la primera pregunta de valoración contingente

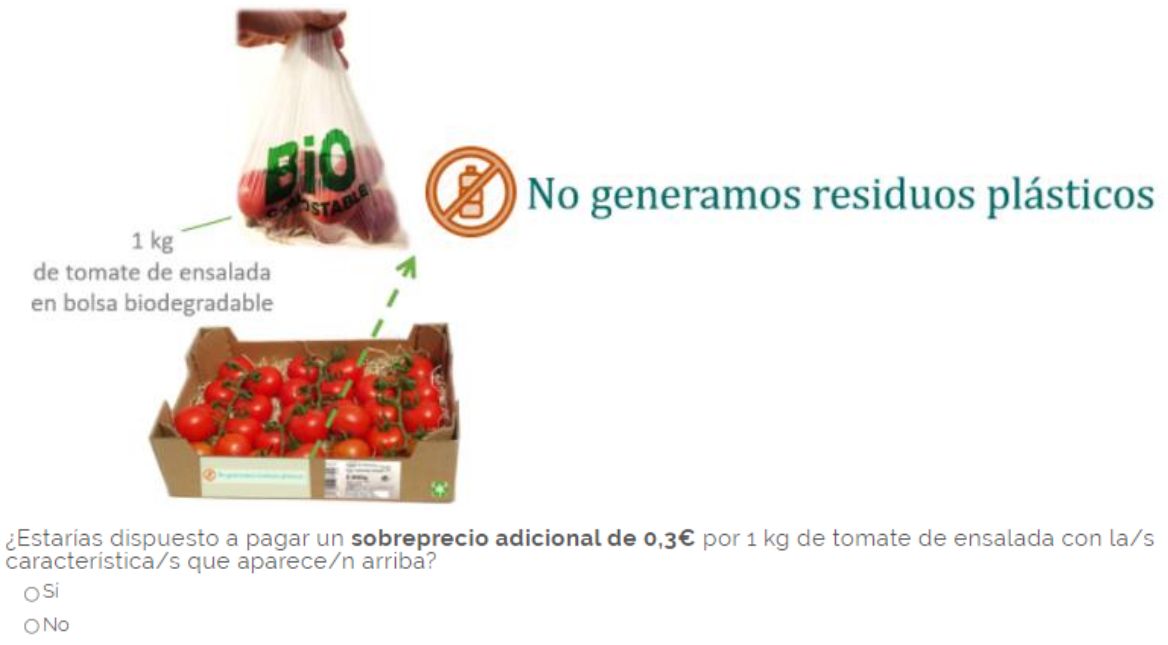

Cada entrevistado contestó a tres pares de estas dos preguntas de valoración contingente, dos para productos que solo incluían un atributo ( "No generamos residuos plásticos", como el ejemplo mostrado en el Cuadro 1) y una referida al producto incluyendo los siete atributos considerados (que se recogen en la Tabla 1, donde se muestra la información proporcionada al entrevistado). Tanto el orden entre las preguntas con uno y todos los atributos, como la selección de los atributos por entrevistado se realizó de forma aleatoria. Antes de empezar el ejercicio de valoración con los tres pares de preguntas, se incluyó el siguiente texto introductorio:

"A continuación, te vamos a presentar una serie de elecciones donde se presentan distintos productos de tomate de ensalada (1 kg, portado en bolsa biodegradable) producidos en Andalucía. Cada elección es independiente, solo debes pensar en el producto que se presenta en cada pantalla. No debes olvidar que dispones de un presupuesto limitado. Por lo tanto, si el precio de las opciones planteadas es mayor de lo que estarías dispuesto a pagar por comprar tomate de ensalada, debes elegir la opción de no compra"

La encuesta se realizó on-line utilizando un panel de consumidores. El cuestionario constó de cuatro bloques: introducción del concepto de BEC y descripción de los atributos; ejercicio de valoración contingente; ejercicio de valoración mejor-peor; características socioeconómicas, de actitud y opinión del entrevistado. Los dos ejercicios de valoración se realizaron de forma aleatorizada. Para la selección de la muestra se aseguró la representatividad de la población adulta de Andalucía usando cuotas de género, edad y por provincias. La muestra se ejecutó en marzo de 2021, obteniéndose un total de 1248 cuestionarios completos. Previamente se empleó un pre-test con cerca de un centenar de entrevistados (incluidos en la muestra final) para testar la correcta compresión del cuestionario. 
Tabla 1. Atributos relacionados con la BEC incluidos en el ejercicio de valoración contingente

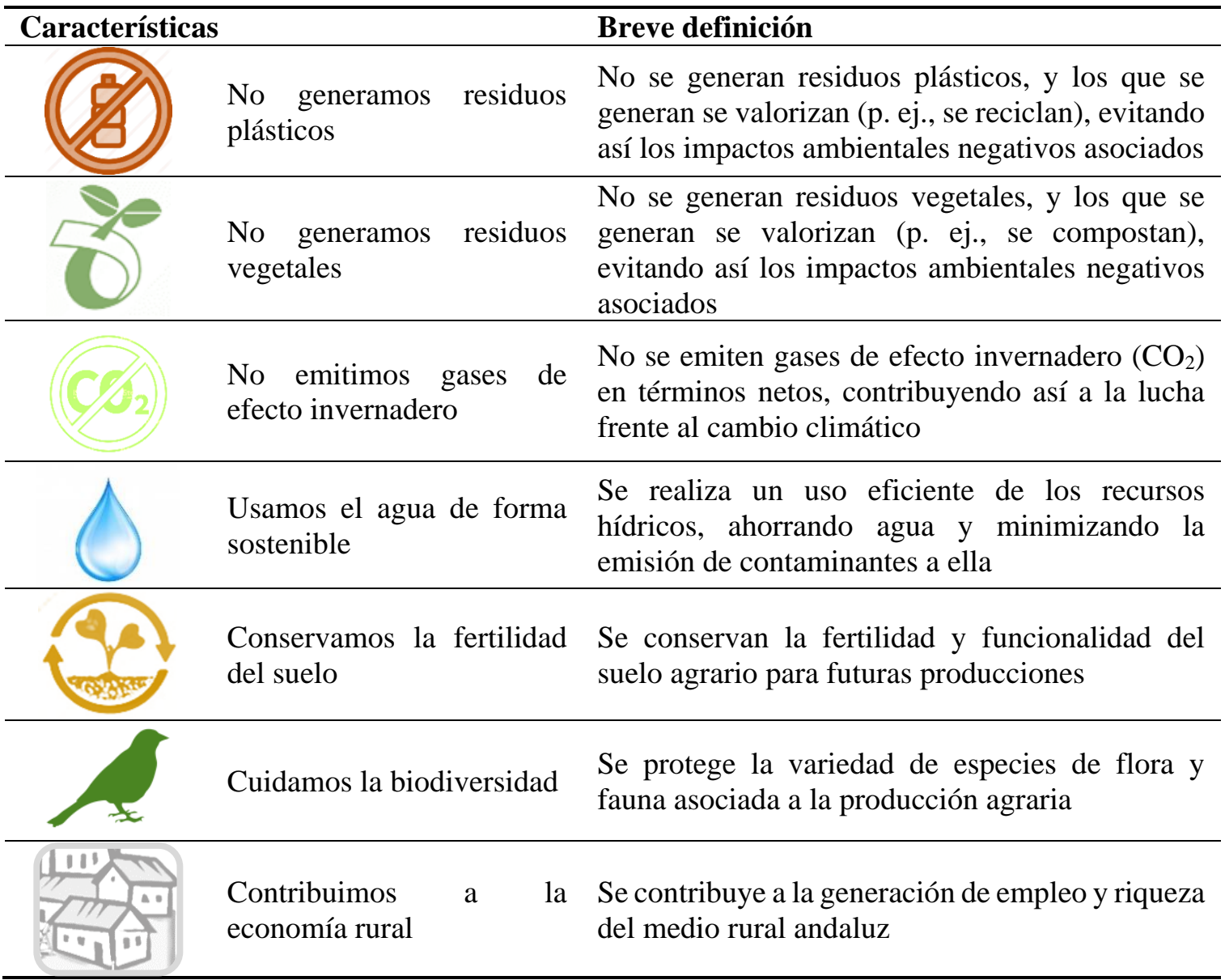

\section{Resultados}

Los resultados preliminares muestran que el $87 \%$ de los consumidores andaluces están dispuestos a pagar un sobreprecio por tomate de ensalada que presente algún atributo relacionado con la BEC. En concreto, para el total de la muestra, el sobreprecio máximo (i.e. extraído de la pregunta semi-abierta de valoración contingente) que en promedio estarían dispuestos a pagar los entrevistados es de $0,52 € / \mathrm{kg}$ de tomate de ensalada por productos que presenten los siete atributos considerados. Esto supone un $37 \%$ más del precio medio que pagan por el tomate de ensalada, el cual según lo recogido en la encuesta es de $1,41 € / \mathrm{kg}$. No obstante, estos resultados preliminares parecen indicar que este sobreprecio varía según el número de atributos (en el caso analizado, uno o siete) y el tipo. Ello se puede comprobar en el Gráfico 1, donde están representados las DAP máximas declaradas por los entrevistados distinguiendo por atributo.

Gráfico 1. Promedio de DAP máxima por atributo incluido en tomate de ensalada

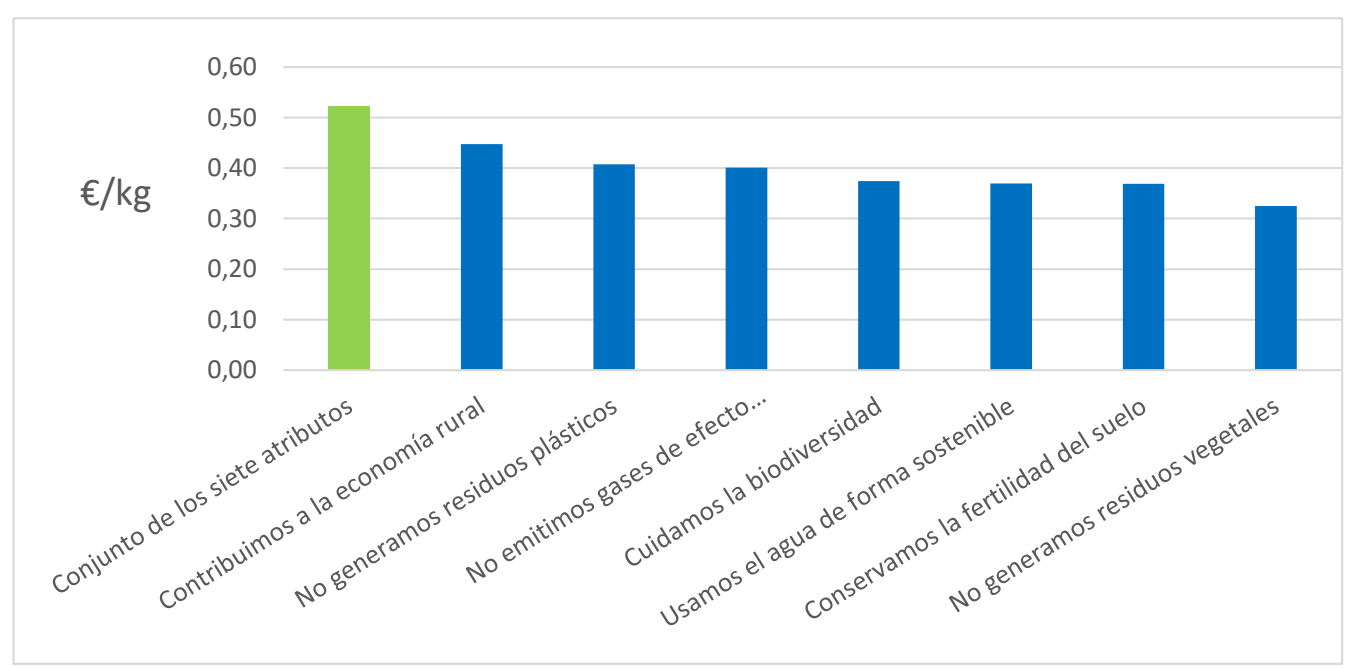


Según se muestra en el Gráfico 1, los consumidores están dispuestos a pagar un sobreprecio por cualquiera de los siete atributos considerados, en todo caso dentro del rango de 0,33-0,45 €/kg de tomate de ensalada. Sin embargo, se observan valores de sobreprecio superiores para los atributos "Contribución a la economía rural", "No generación de residuos plásticos" y "No emitimos gases de efecto invernadero" (todos ellos con DAP máxima de al menos $0,40 € / \mathrm{kg}$ de media), seguidos de "Cuidamos la biodiversidad", "Usamos el agua de forma sostenible" y "Conservamos la fertilidad del suelo" (en el entorno de los 0,37 €/kg) y "No generamos residuos vegetales" (con $0,33 € / \mathrm{kg}$ ). Si se comparan estos resultados con el sobreprecio estimado para el tomate de ensalada que presenta conjuntamente los siete atributos considerados con los obtenidos para el mismo producto con solo un atributo, se puede inferir que el sobreprecio que los consumidores están dispuestos a pagar no sigue una función estrictamente aditiva de los atributos, en la medida que el sobreprecio que pagarían por el conjunto de atributos considerados $(0,52 € / \mathrm{kg})$ es netamente inferior a la suma de los sobreprecios que pagarían por separado. No obstante, estos resultados deben tomarse con precaución, habida cuenta de que se trata de resultados preliminares y que se obtienen de un diferente número de entrevistados: la muestra completa, i.e 1248 individuos para el caso la pregunta de valoración contingente relativa al producto que incorpora el "Conjunto de los siete atributos" y submuestras de 355 a 357, según el atributo, para el caso de las preguntas de valoración contingente de productos con solo un atributo. En cualquier caso, de confirmarse dichos sobreprecios, estos deberían cotejarse con el aumento de costes asociados al empleo de prácticas innovadoras y sostenibles en la horticultura protegida andaluza [una variedad de estas se pueden consultar en Egea et al. (2018) y Sayadi-Gmada et al. (2019)].

\section{Conclusiones}

El desarrollo de la bioeconomía circular en España pasa necesariamente por que el sector agroalimentario adopte sus principios, haciendo de este un sector económica, social y ambientalmente sostenible en el largo plazo. Resulta especialmente relevante conocer las preferencias del consumidor para poder incorporar a los productos alimentarios atributos relacionados con la BEC que son a su vez demandados por los consumidores. El presente estudio analiza las preferencias de los consumidores por productos alimentarios que incluyen dichos atributos, para el caso de estudio de tomate de ensalada en Andalucía. En él se emplean técnicas de preferencias declaradas en una muestra de 1248 consumidores. Los resultados indican que los consumidores están dispuestos a pagar un sobreprecio por cualquiera de los siete atributos considerados, en todo caso dentro del rango de $0,33-0,45 € / \mathrm{kg}$. De mayor a menor sobreprecio, los atributos se ordenan de la siguiente forma: "Contribución a la economía rural", "No generación de residuos plásticos", "No emitimos gases de efecto invernadero", "Cuidamos la biodiversidad", "Usamos el agua de forma sostenible", "Conservamos la fertilidad del suelo" y "No generamos residuos vegetales". Se observa asimismo cómo el sobreprecio que están dispuestos a pagar no sigue una función estrictamente aditiva de los atributos, dado que el sobreprecio que pagarían por el conjunto de atributos considerados es de 0,52 $€ / \mathrm{kg}$ de media. De confirmarse estas estimaciones preliminares, estos resultados sugerirían que el uso de estrategias de diferenciación en el sector del tomate podría aumentar su rentabilidad.

\section{Agradecimientos}

Los datos empleados en este estudio provienen de un contrato realizado desde la Secretaría General de Agricultura, Ganadería y Alimentación de la Consejería de Agricultura, Ganadería, Pesca y Desarrollo Sostenible de la Junta de Andalucía, dentro del proyecto europeo POWER4BIO (https://power4bio.eu/; GA 818351).

\section{Referencias}

Carus, M. y Dammer, L. (2018). "The circular bioeconomy-concepts, opportunities, and limitations". Industrial biotechnology, 14(2): 83-91.

EC (European Commission). (2018). A sustainable bioeconomy for Europe: Strengthening the connection between economy, society and the environment. COM(2018) 673 final. European Commission, Brussels.

Egea, F.J., Torrente, R.G. y Aguilar, A. (2018). "An efficient agro-industrial complex in Almería (Spain): Towards an integrated and sustainable bioeconomy model". New Biotechnology, 40(Pt A): 103-112.

Ketelsen, M., Janssen, M. y Hamm, U. (2020). "Consumers' response to environmentally-friendly food packaging - A systematic review". Journal of Cleaner Production, 254: 120123.

Kirchherr, J., Reike, D. y Hekkert, M. (2017). "Conceptualizing the circular economy: An analysis of 114 definitions". Resources, Conservation and Recycling, 127: 221-232.

Li, S. y Kallas, Z. (2021). "Meta-analysis of consumers' willingness to pay for sustainable food products". Appetite, 163: 105239

Mesías Díaz, F.J., Martínez-Carrasco Pleite, F., Martínez Paz, J.M. y Gaspar García, P. (2012). “Consumer knowledge, consumption, and willingness to pay for organic tomatoes". British Food Journal, 114(3): 318-334.

Sayadi-Gmada, S., Rodríguez-Pleguezuelo, C.R., Rojas-Serrano, F., Parra-López, C., Parra-Gómez, S., García-García, M.d.C., . . . Manrique-Gordillo, T. (2019). "Inorganic waste management in greenhouse agriculture in Almeria (SE Spain): Towards a circular system in intensive horticultural production". Sustainability, 11(14): 3782 . 